\title{
COMPARAÇÃO DA ATIVIDADE FÍSICA E SATISFAÇÃO COM A VIDA EM IDOSOS INSTITUCIONALIZADOS E DOS CENTROS DIA
}

Ananda Siqueira

Graduada em Educação Física. Centro Universitário Metropolitano de Maringá (UNIFAMMA), Brasil.

\section{Thalita Michelle Minella Ribeiro}

Graduada em Educação Física. Centro Universitário Metropolitano de Maringá (UNIFAMMA), Brasil.

\section{Mateus Dias Antunes}

Doutorando em Ciências da Reabilitação na Universidade de São Paulo (USP), Brasil.

\section{José Roberto A. do Nascimento Júnior}

Doutor em Educação Física. Universidade Federal do Vale do São Francisco (UNIVASF), Brasil.

\section{Paolo Marcello da Cunha Fabro}

Doutorando em Educação Física. Universidade Estadual de Londrina (UEL), Brasil.

\section{Letícia Décimo Flesch}

Doutora em Gerontologia. Universidade Estadual de Campinas (UNICAMP), Brasil.

\section{Daniel Vicentini Oliveira}

Doutor em Gerontologia (UNICAMP). Centro Universitário Metropolitano de Maringá (UNIFAMMA) Brasil.
RESUMO: É de grande importância entender a relação entre atividade física e comportamento sedentário com a satisfação com a vida de idosos. O objetivo deste estudo foi comparar a atividade física e a satisfação com a vida entre idosos institucionalizados e idosos usuários dos Centro Dia. Trata-se de estudo transversal, realizado entre fevereiro a junho de 2017, com 31 idosos de ambos os sexos, sendo 19 institucionalizados e 11 frequentadores de Centro Dia, do município de Maringá, Paraná. Foi utilizado um questionário com questões sociodemográficas, Escala de Satisfação com a vida e o International Physical Activity Questionnaire (IPAQ), versão curta. Para a análise dos dados, foi utilizado o teste de Shapiro-Wilk, Qui-quadrado, exato de Fisher, "U" de Mann-Whitney e o coeficiente de correlação de Spearman. A significância adotada foi de $p<0,05$. Os idosos frequentadores dos Centro Dia caminham mais dias por semana $(\mathrm{p}=0,001)$, praticaram mais dias de atividades moderadas por semana $(p=0,039)$ e mais minutos por dia $(p=0,039)$, por semana $(p=0,032)$ destas atividades. O tempo sentado durante a semana apresentou correlação significativa $(\mathrm{p}<0,05)$ e negativa como os minutos de caminhada por dia, os minutos de caminhada por semana e a satisfação com a vida. Já o tempo sentado durante o fim de semana apresentou correlação significativa $(\mathrm{p}<0,05)$ e negativa apenas com os minutos de caminhada por dia. Idosos institucionalizados são menos ativos fisicamente, apesar de ficarem menos tempo sentados durante os fins de semana. Além disso, o comportamento sedentário do idoso é um fator que pode estar relacionado à redução de atividades físicas leves e da satisfação com a vida.

PALAVRAS-CHAVE: Atividade motora; Envelhecimento; Institucionalização.

\section{COMPARING PHYSICAL ACTIVITIES AND LIFE SATISFACTION IN ELDERLY PEOPLE IN INSTITU- TIONS AND DAY CARE CENTERS}

\begin{abstract}
It is highly relevant to understand the relationship between physical activity and sedentary behavior with elderly people's life satisfaction. Physical activity and life satisfaction between elderly people in institutions and elderly ones in day care centers are compared. Current transversal study was undertaken with 31 elderly people of both genders between February and June 2017. Nineteen elderly people were in institutions and 11 were kept in day care centers, in Maringá PR Brazil. A sociodemographic questionnaire, Life Satisfaction Scale and abridged International Physical Activity Questionnaire (IPAQ) were employed. Data analyses employed Shapiro-Wilk test, Chi-square test, Fisher's Exact test, Mann-Whitney U test and Spearman's correlation coefficient, at $p<0.05$. Elderly people from day centers walk more days per week $(p=0.001)$, practice more days of the week in moderate activities $(\mathrm{p}=0.039)$ and more minutes per day $(\mathrm{p}=0.039)$, week
\end{abstract}


$(p=0.032)$ in these activities. Sitting periods during the week provided significant co-relationship $(\mathrm{p}<0.05)$ and negative co-relationship as minutes walking per day, minutes walking per week and life satisfaction. Sitting time during the weekend had a significant co-relationship ( $\mathrm{p}$ $<0.05$ ) and negative co-relationship only with walking minutes per day. Institutionalized elderly people are less active physically in spite of the fact that they spend less time sitting during the weekend. Sedentary behavior of the elderly is a factor that may be related to a decrease in light physical activities and life satisfaction.

KEY WORDS: Motor activity; Aging; Institutionalization.

\section{INTRODUÇÃO}

No processo de envelhecimento humano há um aumento da vulnerabilidade para doenças crônicas não transmissíveis ${ }^{1,2}$, que, por sua vez, têm grande impacto na funcionalidade desses idosos. Quanto maior o número de doenças presentes, maior é o risco de limitações funcionais e incapacidades ${ }^{3}$. A promoção de atividade física para idosos pode ser uma estratégia efetiva de prevenção para o declínio físico e cognitivo, o isolamento social e proteção contra demências ${ }^{4}$. Além disso, idosos que praticam atividade física apresentam maior probabilidade de reportar maior bem-estar subjetivo (BES) ${ }^{5}$.

O BES é a avaliação subjetiva da própria vida e os fatores que interferem nessa avaliação. Ele possui um componente cognitivo (satisfação com a vida), que representa um julgamento cognitivo de domínios da vida, e um emocional (afetos positivo e negativo), que são estados positivos e negativos transitórios. O alto nível de BES se refere à predominância de eventos considerados positivos sobre os negativos ${ }^{6-8}$.

Não é possível avaliar o BES por medidas externas, uma vez que este conceito se refere à percepção do indivíduo sobre a sua própria vida, envolvendo seus valores, metas, experiências e expectativas ${ }^{7,9}$. A satisfação com a vida pode ser compreendida como um julgamento cognitivo consciente, no qual a pessoa compara a sua vida com um padrão ou conjunto de padrões almejados, conduzindo, assim, para uma avaliação global da vida ${ }^{10}$. A satisfação com a vida da velhice está associada a fatores sociodemográficos, físicos, psicológicos e cognitivos ${ }^{11-14}$.
Diante disso, é de grande importância entender a relação entre a atividade física e o comportamento sedentário e como esses fenômenos podem impactar positiva ou negativamente o BES e a satisfação com a vida dessa população idosa. Um melhor entendimento dessas relações pode proporcionar ao profissional atuante na área uma melhor capacidade para propor atividades para essa população e assim podendo melhorar o BES desse público que necessita de cuidados especiais.

Diante disso, é importante também identificar o contexto no qual o idoso está inserido. Neste estudo, foram pesquisados idosos que frequentam os Centro Dia, além de idosos residentes em Instituições de Longa Permanência (ILPI). O Centro Dia caracteriza-se por ser um espaço para atender idosos que possuem limitações para a realização das Atividades de Vida Diária (AVD), que convivem com suas famílias, porém, não dispõem de atendimento de tempo integral no domicílio ${ }^{15}$. Já as ILPI são instituições governamentais ou não governamentais, de caráter residencial, destinadas a domicílio coletivo de pessoas com idade igual ou superior a 60 anos, com ou sem suporte familiar, em condição de liberdade e dignidade e cidadania ${ }^{16-18}$.

Diante da escassez de estudos de alta confiabilidade sobre a atividade física, comportamento sedentário e, principalmente, a satisfação com a vida de idosos usuários dos Centro Dia e institucionalizados, justifica-se a realização deste estudo. A hipótese deste estudo é de que a atividade física e satisfação com a vida sejam menores em idosos institucionalizados quando comparado aos que participam de Centro Dia. Diante disso, o objetivo deste estudo foi comparar a atividade física e a satisfação com a vida entre idosos institucionalizados e idosos usuários dos Centro Dia, além de verificar as relações entre a prática de atividade física e a satisfação com a vida.

\section{METODOLOGIA}

Trata-se de estudo quantitativo, transversal e observacional, realizado entre fevereiro a junho de 2017.

A amostra, escolhida por conveniência e de forma intencional, se constituiu de 31 idosos (60 anos ou mais) com média de idade de 75,13 \pm 8,13 anos, de am- 
bos os sexos, sendo 19 institucionalizados e 11 frequentadores de Centro Dia, do município de Maringá, Paraná. Foram pesquisadas sete ILPI e quatro Centro Dia, totalizando 11 locais.

Foram inclusos na pesquisa idosos com a marcha preservada e/ou que faziam uso de dispositivos auxiliares de marcha (bengala, andador), e que estivessem devidamente cadastrados em um dos Centro Dia ou ILPI do município. Os idosos institucionalizados eram moradores das próprias ILPI, e os idosos dos Centro dia iam a estes locais durante o dia, e retornavam a noite para seus domicílios. Em ambos os locais, havia uma equipe multidisciplinar composta por médicos, enfermeiros, fisioterapeutas, profissionais de educação física, nutricionistas, assistentes sociais, dentre outros.

Foram excluídos os idosos diagnosticados com doenças neuro-degenerativas com comprometimento cognitivo, como Doença de Alzheimer, e aqueles com possível déficit cognitivo, avaliado por meio do Mini Exame do Estado Mental (MEEM).

O MEEM é composto por questões agrupadas em sete categorias: orientação temporal, orientação espacial, registro de três palavras, atenção e cálculo, recordação das três palavras, linguagem e capacidade viso construti$\mathrm{va}^{19}$. As notas de corte utilizadas para exclusão pelo MEEM foram: 17 para os analfabetos; 22 para idosos com escolaridade entre 1 e 4 anos; 24 para os com escolaridade entre 5 e 8 anos e 26 os que tiverem 9 anos ou mais anos de escolaridade. Estes pontos de corte foram baseados nos critérios de Brucki et al. ${ }^{20}$. Correspondem à média obtida por esses autores para cada faixa de escolaridade, menos um desvio padrão. Idosos classificados abaixo do ponto de corte específico para sua escolaridade foram excluídos.

Para caracterizar o perfil sociodemográfico, de saúde, de institucionalização ou frequência no Centro Dia, foi utilizado um questionário semiestruturado, composto por questões referentes a sexo (masculino; feminino), idade (60 a 69 anos; 70 anos ou mais), raça (branca; negra; outas), escolaridade (não estudou; fundamental incompleto; fundamental completo), tabagismo (nunca fumou; já fumou; fuma atualmente), recebe aposentadoria (sim; não), renda mensal (até 1 salário mínimo; mais de 1 salário mínimo), situação conjugal (com companheiro; sem companheiro), presença de doenças (1 doença; 2 doenças ou mais), histórico de quedas nos últimos 6 meses (sim; não), auto percepção de saúde (boa; regular; ruim), auto percepção corporal (gordo; normal; magro), quantas vezes por semana participa de atividades no Centro Dia/ILPIs ( $1 ; 2$ ou mais), auxílio de acessório para a marcha (sim; não).

Foi aplicada a Escala de Satisfação com a Vida com o objetivo de avaliar o julgamento que as pessoas fazem sobre o quão satisfeitas encontram-se com sua vida. A Escala de Satisfação com a Vida é um instrumento proposto por Diener e colaboradores?. Esta medida se compõe de cinco itens (por exemplo, "Na maioria dos aspectos, minha vida é próxima ao meu ideal"), sendo as respostas graduadas de acordo com uma escala tipo Likert, que vai de $1=$ discordo totalmente a $7=$ concordo totalmente. Esta escala tem o propósito de avaliar o julgamento que as pessoas fazem acerca da sua própria satisfação com a vida, sendo elas próprias a eleger, de acordo com seus valores e interesses, os aspectos a serem considerados para expressar tal satisfação. De um possível escore total de 35 pontos, quanto mais próximo do mesmo, melhor a satisfação com a vida do idoso ${ }^{21}$.

A atividade física dos idosos foi avaliada utilizando-se a versão curta do Questionário Internacional de Atividade Física (IPAQ). O mesmo é composto por sete questões abertas e suas informações permitem estimar o tempo despendido, por semana, em diferentes dimensões de atividade física (caminhadas e esforços físicos de intensidades moderada e vigorosa) e de inatividade física (posição sentada). O nível de atividade física foi classificado em sedentário, irregularmente ativo, ativo ou muito ativo. O comportamento sedentário foi avaliado por meio do tempo médio sentado em um dia de semana, e em um dia de final de semana ${ }^{22}$.

Este estudo foi aprovado pelo Comitê de Ética em Pesquisa da Faculdade Metropolitana de Maringá (FAMMA), por meio do parecer 2.302.409/2017. Primeiramente foi realizado contato com o Centro de Referência de Assistência Social (CRAS) de Maringá (PR), para que informassem a lista de ILPIs e Centro Dia que prestam assistência aos idosos no município. Em seguida, foi entrado em contato com o responsável de cada instituição e Centro Dia, explicando a forma que a pesquisa seria realizada. Desse modo, foi entregue um termo de autorização para a participação desses locais na pesquisa, no qual foi assinado pelo responsável dos mesmos.

Foram agendados horários diversos (manhã e 
tarde), de acordo com a disponibilidade dos pesquisadores, tantos para as ILPIs quanto para os Centro Dia para a coleta dos dados. Os idosos que aceitaram participar da pesquisa assinaram o Termo de Consentimento Livre e Esclarecido (TCLE). Aqueles que aceitaram, mas foram incapazes de assinar, fizeram impressão digital no termo.

Para a análise dos dados, foi utilizado frequência e percentual para as variáveis categóricas. Para as variáveis numéricas, inicialmente foi verificada a normalidade dos dados por meio do teste de Shapiro-Wilk. Como os dados não apresentaram distribuição normal, foram utilizadas Mediana (Md) e Quartis (Q1; Q3) para a caracterização dos resultados. Na estatística inferencial, o teste do Qui-quadrado, e quando necessário o teste exato de Fisher, foram empregados para investigar diferenças proporcionais na prevalência das variáveis sociodemográficas e de saúde entre os idosos institucionalizados e frequentadores dos Centro Dia. Na comparação do nível de atividade física e satisfação com a vida entre os grupos, foi utilizado o teste "U" de Mann-Whitney. Calculou-se também o tamanho do efeito $(d)$ por meio do modelo proposto por Cohen ${ }^{23}$ para diferenças dos valores de dois grupos independentes. De acordo com os critérios de Cohen, um valor $d=0,20$ representa tamanho de efeito pequeno; $d=0,50$, médio; e $d=0,80$, grande. Para analisar a correlação entre as variáveis, efetuou-se o coeficiente de correlação de Spearman. A significância adotada foi de $\mathrm{p}$ $<0,05$. Todas as análises foram realizadas no software Statistical Package for the Social Sciences SPSS v.22.0.

\section{RESULTADOS}

Ao analisar o perfil sóciodemográfico dos idosos institucionalizados e frequentadores dos Centro Dia (Tabela 1), houve diferença significativa $(p>0,05)$ nas proporções apenas no sexo $(\mathrm{p}=0,027)$, faixa etária ( $\mathrm{p}$ $=0,005)$ e escolaridade $(p=0,026)$. Tal resultado indica que há uma maior prevalência de idosos do sexo masculino ( $f=14$ ) que são institucionalizados, enquanto nota-se maior prevalência de idosos frequentadores dos Centro Dia acima dos 70 anos $(f=12)$ e que são analfabetos/ fundamental incompleto $(f=12)$. Nas demais variáveis não houve diferença na prevalência das variáveis entre os dois grupos.
Tabela 1. Perfil sóciodemográfico dos idosos institucionalizados e frequentadores dos Centros Dia da cidade de Maringá, Paraná, Brasil

\begin{tabular}{|c|c|c|c|c|}
\hline \multirow[t]{2}{*}{ VARIÁVEIS } & $\begin{array}{c}\text { Institucio- } \\
\text { nalizados } \\
(\mathrm{n}=19)\end{array}$ & $\begin{array}{l}\text { Centros dia } \\
(\mathrm{n}=12)\end{array}$ & \multirow[t]{2}{*}{$\mathbf{X}^{2}$} & \multirow[t]{2}{*}{$\mathbf{P}$} \\
\hline & $f(\%)$ & $f(\%)$ & & \\
\hline \multicolumn{5}{|l|}{ Sexo $^{a}$} \\
\hline Masculino & $14(73,7)$ & $4(33,3)$ & 4,918 & $0,027^{*}$ \\
\hline Feminino & $5(26,3)$ & $8(66,7)$ & & \\
\hline \multicolumn{5}{|l|}{ Estado civil $^{\text {b }}$} \\
\hline C/companheiro & $3(15,8)$ & $1(8,3)$ & 0,364 & 0,493 \\
\hline S/companheiro & $16(84,2)$ & $11(91,7)$ & & \\
\hline \multicolumn{5}{|l|}{ Faixa Etáriab } \\
\hline 60 a 69 anos & $9(47,4)$ & $0(0,0)$ & 8,010 & $0,005^{*}$ \\
\hline 70 anos ou mais & $10(52,6)$ & $12(100,0)$ & & \\
\hline \multicolumn{5}{|l|}{ Renda mensal ${ }^{\mathrm{b}}$} \\
\hline Até 1 salário mínimo & $12(63,2)$ & $8(66,7)$ & 0,040 & 0,577 \\
\hline 1 a 2 salários mínimos & $7(36,8)$ & $4(33,3)$ & & \\
\hline \multicolumn{5}{|l|}{ Escolaridade $^{\mathrm{b}}$} \\
\hline $\begin{array}{l}\text { Analfabeto/Fund. In- } \\
\text { completo }\end{array}$ & $12(63,2)$ & $12(100,0)$ & 5,711 & $0,026 *$ \\
\hline Fundamental Completo & $7(36,8)$ & $0(0,0)$ & & \\
\hline \multicolumn{5}{|l|}{ Tabagismo $^{\mathrm{b}}$} \\
\hline Nunca fumou & $11(57,9)$ & $9(75,0)$ & 0,940 & 0,452 \\
\hline Fuma/Já fumou & $7(36,8)$ & $2(16,7)$ & & \\
\hline \multicolumn{5}{|l|}{$\operatorname{Raça}^{b}$} \\
\hline Branca & $15(78,9)$ & $9(75,0)$ & 0,066 & 0,798 \\
\hline Negra/Outras & $1(5,3)$ & $2(16,7)$ & & \\
\hline \multicolumn{5}{|l|}{ Aposentadoria $^{\mathrm{b}}$} \\
\hline Sim & $4(21,1)$ & $3(25,0)$ & 0,066 & 0,565 \\
\hline Não & $15(78,9)$ & $9(75,0)$ & & \\
\hline
\end{tabular}

Já em relação ao perfil de saúde dos idosos (Tabela 2), verificou-se diferença na prevalência nas variáveis de frequência de atividades $(\mathrm{p}=0,012)$ e nível de atividade física, evidenciando que há maior prevalência de idosos institucionalizados irregularmente ativos/sedentários $(f=17)$ e que mais idosos frequentadores dos Centro Dia realizam atividades físicas no mínimo duas vezes por semana ( $f=12$ ). Nas demais variáveis não houve diferença na prevalência das variáveis entre os dois grupos. 
Tabela 2. Perfil de saúde dos idosos institucionalizados e frequentadores dos centros dia da cidade de Maringá, Paraná, Brasil

\begin{tabular}{|c|c|c|c|c|}
\hline \multirow[t]{2}{*}{ VARIÁVEIS } & $\begin{array}{l}\text { Institucionalizados } \\
\qquad(\mathrm{n}=19)\end{array}$ & $\begin{array}{l}\text { Centros dia } \\
\qquad(\mathrm{n}=12)\end{array}$ & \multirow[t]{2}{*}{$\mathbf{X}^{2}$} & \multirow[t]{2}{*}{$\mathbf{P}$} \\
\hline & $f(\%)$ & $f(\%)$ & & \\
\hline \multicolumn{5}{|l|}{ Percepção de saúde ${ }^{\mathrm{a}}$} \\
\hline Ruim/Regular & $11(57,9)$ & $6(50,0)$ & 0,185 & 0,667 \\
\hline Boa & $8(42,1)$ & $6(50,0)$ & & \\
\hline \multicolumn{5}{|l|}{ Histórico de quedas ${ }^{b}$} \\
\hline Sim & $8(42,1)$ & $1(8,3)$ & 2,597 & 0,101 \\
\hline Não & $11(57,9)$ & $11(91,7)$ & & \\
\hline \multicolumn{5}{|l|}{ Quantidade de doenças $^{\mathrm{a}}$} \\
\hline 1 doença & $11(57,9)$ & $6(50,0)$ & 0,185 & 0,667 \\
\hline 2 ou mais doenças & $8(42,1)$ & $6(50,0)$ & & \\
\hline \multicolumn{5}{|l|}{ Percepção corporal ${ }^{b}$} \\
\hline Gordo & $6(31,6)$ & $4(33,3)$ & 0,010 & 0,611 \\
\hline Normal/Magro & $13(68,4)$ & $8(66,7)$ & & \\
\hline \multicolumn{5}{|l|}{ Tempo de frequência ${ }^{b}$} \\
\hline Até 6 meses & $4(21,1)$ & $4(33,3)$ & 0,579 & 0,676 \\
\hline Mais de 6 meses & $15(78,9)$ & $8(66,7)$ & & \\
\hline \multicolumn{5}{|l|}{ Frequência de atividades ${ }^{\mathrm{b}}$} \\
\hline 1 vez na semana & $8(42,1)$ & $0(0,0)$ & 6,810 & $0,012 *$ \\
\hline 2 vezes ou mais & $11(57,9)$ & $12(100,0)$ & & \\
\hline \multicolumn{5}{|l|}{ Nível de atividade física ${ }^{b}$} \\
\hline Ativo & $2(10,5)$ & $8(66,7)$ & 10,608 & $0,002 *$ \\
\hline Irreg. Ativo/Sedentário & $17(89,5)$ & $4(33,3)$ & & \\
\hline \multicolumn{5}{|l|}{ Auxílio para a marcha ${ }^{b}$} \\
\hline Sim & $1(5,3)$ & $3(25,0)$ & 2,549 & 0,272 \\
\hline Não & $18(94,7)$ & $9(75,0)$ & & \\
\hline
\end{tabular}

*Associação significativa $(\mathrm{p}<0,05)$ - Teste de Qui-quadrado ou Exato de Fisher ${ }^{\mathrm{b}}$.

Ao comparar o nível de atividade física, o comportamento sedentário e a satisfação com a vida dos idosos institucionalizados e frequentadores dos Centro Dia (Tabela 3), foi encontrada diferença significativa entre os grupos nos dias de caminhada por semana $(\mathrm{p}=$ $0,001)$, dias de atividade física moderada por semana ( $\mathrm{p}$ $=0,039)$, minutos de atividade física moderada por dia ( $\mathrm{p}=0,039)$, minutos de atividade física moderada por semana $(p=0,032)$ e tempo sentado em fim de semana $(p=0,001)$. 
Tabela 3. Comparação do nível de atividade física e satisfação com a vida dos idosos institucionalizados e frequentadores dos Centros Dia da cidade de Maringá, Paraná, Brasil

\begin{tabular}{l|c|c|c|c}
\hline \multirow{2}{*}{ Nível de atividade física } & Institucionalizados $(\mathbf{n}=\mathbf{1 9})$ & Centros dias $(\mathbf{n}=\mathbf{1 2})$ & P & Tamanho do efeito \\
\cline { 2 - 5 } & Md (Q1-Q3) & Md (Q1-Q3) & & -0.76 \\
\hline Dias de caminhada & $1,0(0,0 ; 2,0)$ & $5,0(3,0 ; 5,0)$ & $\mathbf{0 , 0 0 1 *}$ & 0.12 \\
Min. de caminhada p/dia & $30,0(0,0 ; 60,0)$ & $20,0(15,0 ; 35,0)$ & 0,795 & -0.10 \\
Min. de caminhada p/sem. & $30,0(0,0 ; 120,0)$ & $75,0(60,0 ; 115,0)$ & 0,059 & -0.49 \\
Dias de atividade moderada & $1,0(0,0 ; 1,0)$ & $3,5(0,0 ; 5,0)$ & $\mathbf{0 , 0 3 9 *}$ & -0.46 \\
Min. de atividade moderada p/dia & $20,0(0,0 ; 30,0)$ & $75,0(0,0 ; 120,0)$ & $\mathbf{0 , 0 3 9 *}$ & $\mathbf{0 , 0 3 2 *}$ \\
Min. de atividade moderada p/sem. & $20,0(0,0 ; 30,0)$ & $270,0(0,0 ; 412,5)$ & -0.52 \\
Tempo sentado durante a semana & $600,0(540,0 ; 660,0)$ & $600,0(600,0 ; 780,0)$ & 0,120 & -0.32 \\
Tempo sentado em fim de semana & $540,0(480,0 ; 600,0)$ & $810,0(720,0 ; 900,0)$ & $\mathbf{0 , 0 0 1 *}$ & -0.70 \\
Satisfação com a vida & $26,0(22,0 ; 30,0)$ & $25,5(25,0 ; 28,0)$ & 0,997 & -0.06 \\
\hline
\end{tabular}

*Diferença significativa - $\mathrm{p}<0,05$ (Teste de "U" de Mann-Whitney).

Ressalta-se que os idosos frequentadores dos Centro Dia caminham mais dias por semana, além de praticarem mais dias de atividades moderadas por semana, $\mathrm{e}$ mais minutos por dia e por semana destas atividades em comparação aos idosos institucionalizados. Em contrapartida, os idosos institucionalizados ficam menos tempo sentados durante os fins de semana. A análise do tamanho do efeito demonstrou magnitude de efeito pequena a média. Não houve diferença no grau de satisfação com a vida $(p=0,997)$ entre os idosos dos dois grupos. A Tabela 4 apresenta a correlação entre as variáveis de comportamento sedentário, nível de atividade física e satisfação com a vida.

Para os idosos institucionalizados foram encontradas as seguintes correlações significativas $(p<0,05)$ : tempo sentado durante a semana com dias de caminhada $(\mathrm{r}=-0,69)$, minutos de caminhada por dia $(\mathrm{r}=-0,73)$, minutos de caminhada por semana $(r=-0,72)$ e a satisfação com a vida $(r=-0,37)$; e tempo sentado durante $o$ fim de semana com dias de caminhada $(r=-0,79)$, minutos de caminhada por dia $(\mathrm{r}=-0,79)$ e minutos de caminhada por semana $(r=-0,80)$. Tais resultados parecem indicar que o comportamento sedentário do idoso institucionalizado é um fator que pode estar relacionado fortemente com a redução de atividades físicas leves e de forma fraca com satisfação com a vida.

Para os idosos do Centro Dia foram encontradas as seguintes correlações significativas ( $p<0,05$ ): tempo sentado durante a semana com minutos de caminhada por dia $(r=-0,38)$, minutos de caminhada por semana $(r=-0,50)$ e satisfação com a vida $(r=-0,37)$; e tempo sentado durante o fim de semana com dias de caminhada $(\mathrm{r}=-0,36)$ e minutos de caminhada por semana $(\mathrm{r}=$ $-0,41)$. Estes achados evidenciam que o comportamento sedentário do idoso do Centro Dia é um fator associado de forma fraca à redução de atividades físicas leves e a satisfação com a vida. 
Tabela 4. Correlação entre as variáveis de comportamento sedentário, nível de atividade física e satisfação com a vida dos idosos institucionalizados e dos Centros Dia da cidade de Maringá, Paraná, Brasil

\begin{tabular}{|c|c|c|c|c|c|c|c|c|c|}
\hline \multirow{3}{*}{ Institucionalizados Centro Dia } & \multirow{2}{*}{\multicolumn{2}{|c|}{$\begin{array}{l}\text { Comportamento } \\
\text { Sedentário }\end{array}$}} & \multicolumn{6}{|c|}{ Nível de atividade física } & \multirow{3}{*}{$\begin{array}{c}\text { Satisfação com } \\
\text { a vida } \\
9\end{array}$} \\
\hline & & & & & & & & & \\
\hline & 1 & 2 & 3 & 4 & 5 & 6 & 7 & 8 & \\
\hline 1. Tempo sentado durante a semana & - & $0,89^{*}$ & $-0,69 *$ & $-0,73^{*}$ & $-0,72 *$ & $-0,26$ & $-0,27$ & $-0,27$ & $-0,37^{*}$ \\
\hline 2. Tempo sentado em fim de semana & $0,90 *$ & - & $-0,79 *$ & $-0,79 *$ & $-0,80^{*}$ & $-0,19$ & $-0,19$ & $-0,20$ & $-0,26$ \\
\hline 3. Dias de caminhada & $-0,22$ & $-0,36 *$ & - & $0,96 *$ & $0,99 *$ & $-0,10$ & $-0,10$ & $-0,08$ & 0,21 \\
\hline 4. Minutos de caminhada $\mathrm{p} / \mathrm{dia}$ & $-0,35$ & $-0,26$ & $-0,56^{*}$ & - & $0,99 *$ & 0,02 & $-0,02$ & 0,02 & 0,20 \\
\hline 5. Minutos de caminhada $\mathrm{p} / \mathrm{semana}$ & $-0,50 *$ & $-0,41^{*}$ & 0,02 & $0,70^{*}$ & - & $-0,02$ & $-0,04$ & $-0,01$ & 0,21 \\
\hline 6. Dias de atividade moderada & $-0,30$ & $-0,12$ & $0,56^{*}$ & $-0,31$ & 0,26 & - & $0,98^{*}$ & $0,99 *$ & 0,11 \\
\hline 7. Minutos de atividade moderada $\mathrm{p} / \mathrm{dia}$ & $-0,20$ & $-0,05$ & $0,47^{*}$ & $-0,35^{*}$ & 0,16 & $0,80^{*}$ & - & $0,99 *$ & 0,11 \\
\hline $\begin{array}{l}\text { 8. Minutos de atividade moderada } \mathrm{p} / \\
\text { semana }\end{array}$ & $-0,28$ & $-0,06$ & $0,36^{*}$ & $-0,21$ & 0,32 & $0,94 *$ & $0,78^{*}$ & - & 0,06 \\
\hline 9. Satisfação com a vida & $-0,38^{*}$ & $-0,22$ & 0,26 & 0,05 & 0,26 & 0,29 & $-0,15$ & 0,20 & - \\
\hline
\end{tabular}

*Correlação Significativa - $\mathrm{p}<0,05$.

\section{DISCUSSÃO}

O objetivo deste estudo foi comparar a atividade física e a satisfação com a vida entre idosos institucionalizados e idosos usuários dos Centro Dia, além de verificar as relações entre a prática de atividade física e a satisfação com a vida. Nesta pesquisa, os idosos institucionalizados mostram-se mais sedentários do que aqueles que frequentavam os Centro Dia. Este dado já era esperado, pois idosos institucionalizados tendem a apresentar maior taxa de sedentarismo ${ }^{24}$.

Nas ILPI brasileiras, encontram-se tanto idosos dependentes como independentes, portanto as instituições devem oferecer $t$ serviços de saúde de maior complexidade para os idosos mais fragilizados, assim como serviços que estimulem a manutenção e promoção da autonomia, independência e participação social dos idosos residentes ${ }^{25}$. A atividade física nas ILPI deve ser estimulada, já que está diretamente relacionada à redução da limitação funcional e à prevenção de doenças crônicas não-transmissíveis ${ }^{26-28}$.

Parece existir também associação entre compor- tamento sedentário e satisfação com a vida. Em estudo canadense com 358 adultos com 55 anos e mais livres de condições médicas e ortopédicas crônicas que podem impedir o treinamento de resistência, a satisfação com a vida foi negativamente associada com tempo sedentário e o tempo sedentário foi preditor mais importante de satisfação do que a realização de treinos de resistência ${ }^{29}$.

A associação entre atividade física e satisfação com a vida em idosos foi constatada em estudos longitudinais. McAuley et al. ${ }^{30}$ realizaram estudo prospectivo com 168 mulheres idosas com follow up de 24 meses. A relação entre atividade física e satisfação com a vida foi indireta, com a autoeficácia e saúde física e mental desempenhando papéis intermediários nessa relação.

Resultado parecido foi observado em estudo de intervenção com 174 idosos, inicialmente sedentários ${ }^{31}$. Após um ano, a atividade física teve efeitos diretos significativos na autoeficácia, autoestima física e afeto positivo. Por sua vez, a autoeficácia e o afeto positivo tiveram efeitos diretos na satisfação com a vida. Ao longo de quatro anos, o aumento da atividade física foi associado à melhoria da autoestima física e do afeto positivo, e as melhorias 
no afeto tiveram um efeito direto na satisfação com a vida. No entanto, estudo com 228 idosos da comunidade no Reino Unido identificou que a satisfação com a vidase mostrou associada com passos por dia, mas não com tempo sedentário ${ }^{32}$.

Uma limitação que deve ser apontada é o tamanho pequeno da amostra estudada, porém, a mesma retrata todos os idosos frequentadores destes locais. Mesmo a pesquisa tendo sido realizada em todas as ILPIs e Centro Dia do município de Maringá (PR), os dados refletem a situação apenas de uma única região brasileira, o que não pode ser generalizada para o perfil dos idosos da nação. Além disso, o desenho observacional do estudo impede inferir causalidade. Um dos principais pontos fortes da presente pesquisa se refere às diferenças no comportamento para a prática de atividade física entre idosos que vivem em instituições de longe permanência e idosos que apenas se deslocam para o centro de convivência para realizas atividades físicas e culturais, evidenciando que este fator parece interferir no comportamento do idoso.

Os dados desta pesquisa sugerem que maiores níveis de atividade física e uma vida menos sedentária podem afetar a forma como o idoso percebe e avalia a sua vida, seja no contexto da do Centro Dia ou da ILPI.

\section{CONCLUSÃO}

Conclui-se que existe maior prevalência de idosos institucionalizados irregularmente ativos/sedentários e que mais idosos frequentadores dos Centro Dia realizam atividades físicas no mínimo duas vezes por semana. Ressalta-se que os idosos frequentadores dos Centro Dia caminham mais dias por semana, além de praticarem mais dias de atividades moderadas por semana, e mais minutos por dia e por semana destas atividades em comparação aos idosos institucionalizados.

\section{REFERÊNCIAS}

1. Boccolini CS, Camargo AT da SP. Morbimortalidade por doenças crônicas no Brasil: situação atual e futura. Rio de Janeiro: Fundação Oswaldo Cruz; 2016. 25p. (Textos para Discussão).

2. Mino-León D, Reyes-Morales H, Doubova SV, Pérez-Cuevas R, Giraldo-Rodríguez L, Agudelo-Botero M. Multimorbidity Patterns in Older Adults: An Approach to the Complex Interrelationships Among Chronic Diseases. Arch Med Res. janeiro de 2017; 48 (1): 121-7.

3. Stenholm S, Westerlund H, Head J, Hyde M, Kawachi I, Pentti J, et al. Comorbidity and Functional Trajectories From Midlife to Old Age: The Health and Retirement Study. J Gerontol A Biol Sci Med Sci. março de 2015; 70 (3): 332-8.

4. WHO. Who Global Forum on Innovations for Ageing Populations. 2014.

5. Ku P-W, Fox KR, Liao Y, Sun W-J, Chen L-J. Prospective associations of objectively assessed physical activity at different intensities with subjective well-being in older adults. Qual Life Res. novembro de 2016; 25 (11): 2909-19.

6. Almeida LM, Pereira HP, Fernandes HM. Efeitos de diferentes tipos de prática desportiva no bem-estar psicológico de jovens estudantes do ensino profissional. Rev Iberde Psicol Ejerc Dep. 2018; 13 (1): 15-21.

7. Lima LMM, Santos CSVDB, Almeida MCBMD, Martins MMFPD Ajustamento psicossocial e saúde em idosos: análise de clusters. Revista de Enfermagem Referência. 2008; 1 (16) 9-18.

8. Parente LF, Cunha M, Galhardo A, Couto M. Autocompaixão, bem-estar subjetivo e estado de saúde na idade avançada. Rev Port Invest Comp Soc. 2017; 4 (1): 3-13.

9. Diener E. Subjective well-being. Psychol Bull. maio de 1984; 95 (3): 542-75.

10. Martins E, Fernandes R, Mendes F. Bem-estar subjetivo e atividade física em pessoas idosas. Millenium. 2017; 2 (2): 65-72.

11. Kolosnitsyna M, Khorkina N, Dorzhiev H. Determi- 
nants of Life Satisfaction in Older Russians. Ageing Int. setembro de 2017; 42 (3): 354-73.

12. Ng ST, Tey NP, Asadullah MN. What matters for life satisfaction among the oldest-old? Evidence from China. Quinn TJ, organizador. PLOS ONE. 10 de fevereiro de 2017; 12 (2): e0171799.

13. Karadag Arli S, Bakan AB, Varol E, Aslan G. Investigation of pain and life satisfaction in older adults: Pain and life satisfaction. Geriatr Gerontol Int. janeiro de 2018; 18 (1): 5-11.

14. Delhom I, Gutierrez M, Lucas-Molina B, Meléndez JC. Emotional intelligence in older adults: psychometric properties of the TMMS-24 and relationship with psychological well-being and life satisfaction. Int Psychogeriatr. agosto de 2017; 29 (08): 1327-34.

15. Macêdo LPV, Vieira GÂDCM, Costa MML. Relation between the functional capacity $f$ the elderly and institutionalization: an integrative review. Cuid Fund. 2018; 10 (2): 542-548.

16. Alves MB, Menezes MDR, Felzemburg RDM, Silva VA, Amaral JB. Instituições de longa permanência para idosos: aspectos físico-estruturais e organizacionais. Esc Anna Nery Rev Enferm. 2017; 21 (4): 1-8.

17. Born T, Boechat NS. Qualidade dos cuidados ao idoso institucionalizado. In: Tratado de Geriatria e Gerontologia. $4^{\mathrm{a}}$ ed. Rio de Janeiro: Guanabara Koogan; 2017. p. 1301-6.

18. Sociedade Brasileira de Geriatria e Gerontologia. Seção São Paulo. Instituição de longa permanência para idosos: manual de funcionamento. São Paulo: SBGG; 2003.

19. Folstein M, Folstein S, Mchugh P. Mini Exame do Estado Mental: um método prático para classificar o estado cognitivo dos pacientes para o clínico. J Pesqui Psiquiátrica. 1975; 12.

20. Brucki SMD, Nitrini R, Caramelli P, Bertolucci PHF, Okamoto IH. Sugestões para o uso do mini-exame do estado mental no Brasil. Arq Neuropsiquiatr. setembro de 2003; 61 (3B): 777-81.

21. Gouveia VV, Chaves SS da S, Oliveira ICP de, Dias MR, Gouveia RSV, Andrade PR d. A utilização do QSG-12 na população geral: estudo de sua valida- de de construto. Psicol Teor E Pesqui. dezembro de 2003; 19 (3): 241-8.

22. Matsudo S, Araújo T, Matsudo V, Andrade D, Andrade E, Oliveira LC, et al. Questionário Internacional de Atividade Física (Ipaq): Estudo de Validade e reprodutibilidade no Brasil. Rev Bras Atividade Física Saúde. 2001; 6 (2): 5-18.

23. Cohen J. Quantitative methods in psychology: a power primer. Psychological Bulletin, v. 112, n. 1, p. 155-159, 1992.

24. Marmeleira J, Ferreira S, Raimundo A. Physical activity and physical fitness of nursing home residents with cognitive impairment: A pilot study. Exp Gerontol. dezembro de 2017; 100: 63-9.

25. Fagundes KVDL, Ribeiro ME, Morais Ribeiro JH de, Siepierski CT, Silva JV da, Mendes MA. Entidades de larga permanencia como alternativa para acoger adultos mayores. Rev Salud Pública. $1^{\circ}$ de março de 2017; 19 (2): 210-4.

26. Alves J de C, Freitas EF. Atividade física. In: Freitas EF, Py L, organizadores. Tratado de Geriatria e Gerontologia. Rio de Janeiro: Guanabara Koogan; 2016.

27. Barreto M da S, Carreira L, Marcon SS. Envelhecimento populacional e doenças crônicas: Reflexões sobre os desafios para o Sistema de Saúde Pública. Rev Kairós Gerontol. 2015; 18 (1): 325-39.

28. Mankowski RT, Anton SD, Axtell R, Chen S-H, Fielding RA, Glynn NW, et al. Device-Measured Physical Activity As a Predictor of Disability in Mobility-Limited Older Adults. J Am Geriatr Soc. outubro de 2017; 65(10): 2251-6.

29. Bampton EA, Johnson ST, Vallance JK. Profiles of resistance training behavior and sedentary time among older adults: Associations with health-related quality of life and psychosocial health. Prev Med Rep. 2015; 2: 773-6.

30. McAuley E, Doerksen SE, Morris KS, Motl RW, Hu L, Wójcicki TR, et al. Pathways from Physical Activity to Quality of Life in Older Women. Ann Behav Med. agosto de 2008; 36 (1): 13-20.

31. Elavsky S, McAuley E, Motl RW, Konopack JF, Marquez DX, Hu L, et al. Physical activity enhances long- 
term quality of life in older adults: Efficacy, esteem, and affective influences. Ann Behav Med. outubro de 2005; 30 (2): 138-45.

32. Withall J, Stathi A, Davis M, Coulson J, Thompson J, Fox K. Objective Indicators of Physical Activity and Sedentary Time and Associations with Subjective Well-Being in Adults Aged 70 and Over. Int J Environ Res Public Health. 02 de janeiro de 2014; 11 (1): 643-56.

Aceito em: 01/11/2018

Recebido em: 28/12/2018 